\title{
Predictors of Transition to Hospice Care Among Hospitalized Older Adults With a Diagnosis of Dementia in Texas: A Population-Based Study
}

\author{
Lavi Oud
}

\begin{abstract}
Background: Decedent older adults with dementia are increasingly less likely to die in a hospital, though escalation of care to a hospital setting, often including critical care, remains common. Although hospice is increasingly reported as the site of death in these patients, the factors associated with transition to hospice care during end-of-life (EOL) hospitalizations of older adults with dementia and the extent of preceding escalation of care to an intensive care unit (ICU) setting among those discharged to hospice have not been examined.
\end{abstract}

Methods: We identified hospitalizations aged $\geq 65$ years with a diagnosis of dementia in Texas between 2001 and 2010. Potential factors associated with discharge to hospice were evaluated using multivariate logistic regression modeling, and occurrence of hospice discharge preceded by ICU admission was examined.

Results: There were 889,008 elderly hospitalizations with a diagnosis of dementia during study period, with 40,669 (4.6\%) discharged to hospice. Discharges to hospice increased from 908 (1.5\%) to 7,398 (6.3\%) between 2001 and 2010 and involved prior admission to ICU in $45.2 \%$ by 2010 . Non-dementia comorbidities were generally associated with increased odds of hospice discharge, as were development of organ failure, the number of failing organs, or use of mechanical ventilation. However, discharge to hospice was less likely among nonwhite minorities (lowest among blacks: adjusted odds ratio (aOR): $0.67 ; 95 \%$ confidence interval (CI): $0.65-0.70$ ) and those with noncommercial primary insurance or the uninsured (lowest among those with Medicaid: aOR (95\% CI): $0.41(0.37$ - 0.46)).

Conclusions: This study identified potentially modifiable factors associated with disparities in transition to hospice care during EOL hospitalizations of older adults with dementia, which persisted across comorbidity and severity of illness measures. The prevalent discharge to hospice involving prior critical care suggests that key discussions about goals-of-care likely took place following further escalation of

Manuscript accepted for publication October 13, 2016

Division of Pulmonary and Critical Care Medicine, Department of Internal Medicine, Texas Tech University Health Sciences Center at the Permian Basin, Odessa, TX 79763, USA. Email: lavi.oud@ttuhsc.edu

doi: https://doi.org/10.14740/jocmr2783w care to ICU. Together these findings can inform system- and clinician-level interventions to facilitate timely and consistent use of hospice to meet patients' goals of care.

Keywords: Dementia; Hospice; Hospitalization; Intensive care unit

\section{Introduction}

Hospice care is increasingly used among patients with dementia $[1,2]$, though only a minority are referred, even among those with advanced disease [3]. Although the majority of healthcare proxies (HCP) of patients with advanced dementia believe that comfort support is the primary goal of care [3], escalation of care commonly occurs $[1,4]$. Thus, do-not-hospitalize orders remain uncommon among nursing home residents with dementia [5]. Moreover, decisions to forgo hospitalization are commonly delayed until death is imminent [6], though transitions of care can be associated with increased patient and family burden and with interventions inconsistent with patients' goals of care [7]. The latter findings may explain in part the reported exponential rise in hospitalization of older adults with dementia in the United States [8], with a recent study [9] suggesting that growth in hospitalization volume of these patients in Texas outpaced the estimated growth rate of state population with dementia [10].

Hospitalized patients with dementia represent, by definition, a subpopulation where HCP generally considered such escalation of care appropriate, at least implicitly. Thus, data on factors and barriers associated with subsequent transition to hospice care during end-of-life (EOL) hospitalizations in these patients would inform interventions to better meet patients' goals of care and limit non-beneficial burdensome interventions. However, there have been no reports, to our knowledge, on the determinants of hospice utilization among patients with dementia, once care has been escalated to hospital setting, and transitions to hospice care among hospitalized patients with dementia were generally not addressed [11].

In addition, further escalation of care is common among hospitalized patients with dementia, often including admission to intensive care unit (ICU). We recently reported a rapid growth of in ICU admissions among hospitalized older adults with dementia in Texas, with findings suggesting that these 
patients may account for nearly one in four ICU admissions among older adults in the state by 2020 [9]. However, patients with dementia may be especially vulnerable to accelerated cognitive and physical decline, and with decreased survival following critical illness [12]. Nevertheless, although admissions to ICU can be avoidable when inconsistent with patients' goals of care, such transfers can often occur without preceding discussion about their merit and care alternatives [13]. Finally, although ICU is increasingly used among decedents with dementia in the last 30 days of life [1], there are no data on the extent of transition to hospice care preceded by admission to ICU among hospitalized patients with dementia.

We conducted a population-level examination of hospitalized older adults with a diagnosis of dementia in Texas to identify factors associated with hospice utilization in the inpatient setting, and to quantify temporal patterns of escalation of care to ICU setting preceding discharge to hospice.

\section{Materials and Methods}

\section{Data sources}

We used the Texas Inpatient Public Use Data File (TIPUDF) to perform a retrospective, population-based cohort study of state residents with a diagnosis of dementia. TIPUDF is an administrative data set maintained by the Texas Department of State Health Services [14] and was previously described [15]. Briefly, the data set includes de-identified inpatient discharge data on the demographic, clinical, resource utilization, and outcome domains from non-federal state-licensed hospitals, and captures $93-97 \%$ of all hospital discharges in the state. The Institutional Review Board of Texas Tech Health Sciences Center has determined that the present study is exempt from formal review due to use of publicly available, de-identified data.

\section{Study population}

We used previously described International Classification of Diseases, Ninth Edition, Clinical Modification (ICD-9-CM) codes 290.0-290.4, 291.1, 291.2, 292.82, 292.83, 294.0, 294.1, 294.8, 294.9, 331.0, 331.1, 331.2, 331.7, 331.82, 331.9, 797 $[16,17]$ to identify state residents aged $\geq 65$ years hospitalized with a diagnosis of dementia during the years 2001 - 2010. All individual hospitalizations meeting these criteria were included in the analytic cohort and served as denominator for derived descriptive reports, unless stated otherwise.

\section{Data collection}

We collected data on patients' age, gender, race/ethnicity (categorized as non-Hispanic black (black), non-Hispanic white (white), Hispanic, and other), primary payer health insurance (categorized as commercial, Medicaid, Medicare, no health insurance, and other), non-dementia comorbid conditions (based
Table 1. The Demographics and Health Insurance Characteristics of Older Adult Hospitalizations With a Diagnosis of Dementia, 2001 - 2010

\begin{tabular}{|ll}
\hline Group & $\mathbf{n}=\mathbf{8 8 9 , 0 0 8}$ \\
\hline Age $(\text { years, } \mathrm{n}(\%))^{\mathrm{a}}$ & \\
\hline $65-79$ & $334,226(37.6)$ \\
$\geq 80$ & $554,782(62.4)$ \\
\hline Female $(\mathrm{n}(\%))^{\mathrm{a}, \mathrm{b}}$ & $550,987(64.4)$ \\
Race/ethnicity $(\mathrm{n}(\%))^{\mathrm{a}}$ & \\
\hline White & $590,806(66.5)$ \\
\hline Hispanic & $141,202(15.9)$ \\
\hline Black & $107,746(12.1)$ \\
\hline Other & $47,502(5.3)$ \\
\hline Missing & $2,035(0.2)$ \\
Health insurance $(\mathrm{n}(\%))^{\mathrm{a}}$ & \\
\hline Private & $79,428(8.9)$ \\
\hline Medicare & $774,847(87.2)$ \\
\hline Medicaid & $16,065(1.8)$ \\
\hline Uninsured & $12,971(1.5)$ \\
Other & $5,304(0.6)$ \\
Missing & $393(0.04)$ \\
\hline
\end{tabular}

apercent figures are rounded. ${ }^{b}$ Gender was masked in 33,297 hospitalizations. The denominator used to derive gender percentages for the cohort was based on hospitalizations with available gender designation $(\mathrm{n}=855,711)$.

on the Deyo modification of the Charlson comorbidity index [18]), type of failing organs (Supplementary Table 1, www. jocmr.org), use of mechanical ventilation (ICD-9-CM codes 96.70-96.72), admission to ICU (defined as presence of an intensive care unit charge greater than \$0), in-hospital death and discharge to hospice.

\section{Data analysis}

Because TIPUDF provides discharge-level, rather than patientlevel information, we reported admissions with a diagnosis of dementia as number of hospitalizations. Hospitalization endings with either in-hospital death or discharge to hospice were described as EOL hospitalizations.

Group data are reported as counts and percentages for categorical variables. The Chi-square test was used to compare categorical data. Multivariate logistic regression models were constructed to examine candidate covariates as predictors of discharge to hospice. Covariates were considered for multivariate regression models if they were either statistically significant $(\mathrm{P}<0.10)$ or had odds ratio $(\mathrm{OR}) \geq 1.5$ or $\leq 0.66$ on univariate analysis. Candidate covariates included age, gender, race/ethnicity, health insurance, comorbid conditions, measures of severity of illness (type and number of failing organs) $[19,20]$, and use of mechanical ventilation. Separate models 
Table 2. Comorbid Conditions of Older Adult Hospitalizations With a Diagnosis of Dementia, 2001 - 2010

\begin{tabular}{|ll}
\hline Group & $\mathbf{n}=\mathbf{8 8 9 , 0 0 8}$ \\
\hline Deyo-Charlson index-based comorbidities (n (\%)) & \\
\hline Congestive heart failure & $218,508(24.6)$ \\
\hline Peripheral vascular disease & $104,925(11.8)$ \\
Lung disease & $188,171(21.3)$ \\
Cerebrovascular disease & $139,289(15.7)$ \\
Connective tissue disease & $15,955(1.8)$ \\
Diabetes mellitus & $232,916(26.2)$ \\
Renal disease & $110,728(12.5)$ \\
Liver disease & $17,656(2.0)$ \\
Malignancy & $41,048(4.6)$ \\
HIV infection & $980(0.1)$ \\
\hline
\end{tabular}

were constructed to examine the predictive roles of individual organ failures and number of failing organs. The state of Texas masks gender data of hospitalizations with a diagnosis of infection with the human immunodeficiency virus (HIV), and ethanol or drug abuse. Thus, analyses of the predictive role of gender were restricted to hospitalizations without the aforementioned three diagnoses. Similarly, models that examined the predictive role of HIV infection did not include gender. Because marked rise has been found in use of hospice during study period, which could have been affected by changing roles of the examined predictive covariates, we performed sensitivity analyses, re-examining modeled covariates among hospitalizations with dementia in the years 2001 and 2010 to assess for changes in the direction of association or statistical significance at the start and end of study period. Adjusted odds ratios (aOR) and corresponding 95\% confidence intervals $(95 \% \mathrm{CI})$ were calculated.

All statistical analyses were performed using MedCalc version 15.6.1 (MedCalc Software, Ostend, Belgium) and SAS version 9.3 (SAS Institute, Cary, NC, USA). A two-sided P value $<0.05$ was considered statistically significant.

\section{Results}

There were 889,008 hospitalizations with a diagnosis of dementia during study period. The demographic characteristics, health insurance, comorbid conditions, development of organ failure and use of mechanical ventilation of this cohort are detailed in Tables 1-3. The corresponding data for hospitalizations during the years 2001 and 2010 are outlined in Supplementary Tables 2-4 (www.jocmr.org).

The majority of hospitalizations $(62.4 \%)$ were aged 80 years or older, with about two in three being women. Minorities accounted for 33.3\% of hospitalizations. Medicare insurance was the primary payer program for the vast majority, though $1.5 \%$ lacked health insurance, and $1.8 \%$ had Medicaid. Diabetes (26.2\%) and congestive heart failure (24.6\%) were the most common comorbid conditions. One or more organ
Table 3. Organ Failure and Use of Mechanical Ventilation Among Older Adult Hospitalizations With a Diagnosis of Dementia, 2001 - 2010

\begin{tabular}{|ll}
\hline Group & $\mathbf{n}=\mathbf{8 8 9 , 0 0 8}$ \\
\hline Type of organ failure (n (\%)) & \\
$\quad$ Respiratory & $49,481(5.6)$ \\
Cardiovascular & $40,678(4.6)$ \\
$\quad$ Renal & $77,173(8.7)$ \\
Hepatic & $3,758(0.4)$ \\
\hline Hematological & $26,438(3.0)$ \\
Metabolic & $16,527(1.9)$ \\
Neurological & $66,422(7.5)$ \\
Number of failing organs (n (\%)) & \\
1 & $166,418(18.7)$ \\
2 & $37,556(4.2)$ \\
3 & $9,067(1.0)$ \\
\hline 4 & $2,924(0.3)$ \\
Mechanical ventilation (n (\%)) & $15,373(1.7)$ \\
\hline
\end{tabular}

failures developed in 215,965 (24.3\%) hospitalizations, with renal $(8.7 \%)$, neurological $(7.5 \%)$, and respiratory $(5.6 \%)$ systems being the most commonly affected. Mechanical ventilation was used in $31.1 \%$ of those with respiratory failure.

On examination of patient attributes in 2001 and 2010, the key noted changes were increasing male and Hispanic hospitalizations, rise in use of commercial insurance, higher rates of most examined comorbid conditions and substantial increase in the rates of all types of examined organ failures and the number of failing organs. The use of mechanical ventilation in the cohort increased two-fold.

There were 44,174 (5.0\%) in-hospital deaths and discharge to hospice was reported in 40,669 (4.6\%) hospitalizations, thus accounting for $47.9 \%$ of EOL hospitalizations for the whole cohort. The corresponding data on hospital mortality and discharge to hospice during 2001 and 2010 are reported in Supplementary Table 4 (www.jocmr.org), with 908 (1.5\%) and 7,398 (6.3\%) discharged to hospice, respectively. The latter figures correspond to $19.9 \%$ (2001) and 57.5\% (2010) of EOL hospitalizations. Predictors of discharge to hospice are outlined in Table 4. Hospitalizations aged 80 years or older had increased odds of hospice use, while all examined non-white race groups had lower odds of hospice utilization, being lowest among blacks (aOR (95\% CI): 0.67 (0.65 - 0.70); $\mathrm{P}<0.0001)$. Primary insurance other than commercial also predicted lower transition to hospice care, being lowest among hospitalizations with Medicaid insurance (aOR (95\% CI): 0.41 (0.37 - 0.46); P $<0.0001)$. Hospitalizations with non-dementia comorbidities were generally more likely to have discharge to hospice, with the odds being highest among those with malignancy (aOR (95\% CI): 3.72 (3.61 - 3.84); $\mathrm{P}<0.0001)$.

Use of hospice was increased with each of the examined organ failures and increasing number of failing organs increased the odds of discharge to hospice from 2.02 to 6.18 
Table 4. Logistic Regression Analysis of the Factors Associated With Discharge to Hospice Among Older Adult Hospitalizations With a Diagnosis of Dementia, 2001 - 2010

\begin{tabular}{|c|c|c|c|}
\hline Covariate & Adjusted odds ratio & $95 \%$ confidence interval & $\mathbf{P}$ \\
\hline Age $\geq 80$ years $^{\mathrm{a}}$ & 1.78 & $1.73-1.82$ & $<0.0001$ \\
\hline \multicolumn{4}{|l|}{ Race/ethnicity ${ }^{\mathrm{b}}$} \\
\hline Black & 0.67 & $0.65-0.70$ & $<0.0001$ \\
\hline Hispanic & 0.80 & $0.78-0.82$ & $<0.0001$ \\
\hline Other & 0.81 & $0.78-0.85$ & $<0.0001$ \\
\hline \multicolumn{4}{|l|}{ Health insurance ${ }^{c}$} \\
\hline No health insurance & 0.86 & $0.79-0.94$ & 0.0008 \\
\hline Medicaid & 0.41 & $0.37-0.46$ & 0.0001 \\
\hline Medicare & 0.68 & $0.66-0.70$ & $<0.0001$ \\
\hline Other & 0.68 & $0.59-0.78$ & 0.0001 \\
\hline \multicolumn{4}{|l|}{ Chronic comorbidity } \\
\hline Congestive heart failure & 1.35 & $1.32-1.38$ & $<0.0001$ \\
\hline Peripheral vascular disease & 1.30 & $1.27-1.34$ & 0.0001 \\
\hline Cerebrovascular disease & 1.17 & $1.14-1.20$ & $<0.0001$ \\
\hline Lung disease & 0.90 & $0.87-0.92$ & $<0.0001$ \\
\hline Liver disease & 1.12 & $1.05-1.20$ & 0.0008 \\
\hline Diabetes & 0.78 & $0.76-0.80$ & $<0.0001$ \\
\hline Renal disease & 1.15 & $1.12-1.18$ & $<0.0001$ \\
\hline Malignancy & 3.72 & $3.61-3.84$ & $<0.0001$ \\
\hline HIV infection ${ }^{\mathrm{d}}$ & 1.26 & $0.95-1.68$ & 0.1033 \\
\hline \multicolumn{4}{|l|}{ Type of organ failure } \\
\hline Respiratory & 2.54 & $2.46-2.63$ & $<0.0001$ \\
\hline Cardiovascular & 1.41 & $1.36-1.47$ & $<0.0001$ \\
\hline Renal & 2.13 & $2.07-2.19$ & $<0.0001$ \\
\hline Hepatic & 1.58 & $1.35-1.86$ & $<0.0001$ \\
\hline Hematological & 1.16 & $1.10-1.22$ & $<0.0001$ \\
\hline Metabolic & 1.54 & $1.46-1.62$ & $<0.0001$ \\
\hline Neurological & 1.52 & $1.48-1.57$ & $<0.0001$ \\
\hline \multicolumn{4}{|l|}{ Number of failing organs $\mathrm{e}^{\mathrm{e}}$} \\
\hline 1 & 2.02 & $1.97-2.07$ & $<0.0001$ \\
\hline 2 & 3.46 & $3.33-3.59$ & $<0.0001$ \\
\hline 3 & 4.77 & $4.48-5.07$ & $<0.0001$ \\
\hline$\geq 4$ & 6.18 & $5.61-6.82$ & $<0.0001$ \\
\hline Mechanical ventilation ${ }^{f}$ & 1.12 & $1.06-1.18$ & 0.0001 \\
\hline
\end{tabular}

${ }^{a}$ Age 65 - 79 years was used as referent. bWhite race was used as referent. ${ }^{c}$ Commercial insurance was used as referent. ${ }^{d}$ Human immunodeficiency virus. e Lack of organ failure was used as referent. ${ }^{f}$ Modeled with the number of failing organs.

among those with one and $\geq 4$ organ failures, respectively. Mechanical ventilation was associated with increased use of hospice when modeled with the number of failing organs, being collinear, as expected, with respiratory failure $(\mathrm{r}=0.53)$.

On sensitivity analyses (Supplementary Table 5, www. jocmr.org), generally no changes were noted in the direction of association of patients' demographics or health insurance and hospice discharge between 2001 and 2010. The exceptions have been for gender, showing a non-significant effect and lower odds of hospice discharge among males in 2001 and
2010, respectively, and for those lacking health insurance, with the latter no longer associated with hospice discharge in 2010. Similarly, the association of comorbid conditions, development of specific type of organ failure, or the number of failing organs and discharge to hospice remained mostly unchanged. Exceptions were exclusion on univariate analyses for lung disease, HIV infection (on 2001 and 2010) and liver disease (in 2001), and development of hepatic and metabolic failures in 2001 , with the latter four related in part to small number of hospitalizations. In addition, mechanical ventilation was not 
associated with hospice discharge in 2001.

Admission to ICU occurred in 276,056 hospitalizations (31.1\%). The rate of admission to ICU rose from $21.4 \%$ to $37.9 \%$ between 2001 and $2010(\mathrm{P}<0.0001)$. Discharge to hospice was reported in 16,111 (5.8\%) hospitalizations among those admitted to ICU during study period. The rate of discharge to hospice among ICU admissions increased from $1.7 \%$ to $7.7 \%$ between 2001 and 2010 . Finally, discharges to hospice preceded by ICU admissions accounted for $39.6 \%$ of all hospice discharges in the cohort, with the rate rising from $24.6 \%$ in 2001 to $45.2 \%$ in 2010 .

\section{Discussion}

In this large population-level study, we showed that among hospitalized older adults with a diagnosis of dementia increasing hospice utilization led to its accounting for the majority of EOL hospitalizations by the end of the last decade. Increased transition to hospice care was associated with the presence of non-dementia comorbidities, development of organ failure and the number of failing organs. However, there has been persistent disparate impact of patients' race/ethnicity and type of health insurance on the odds to hospice discharge. In addition, discharges to hospice were increasingly preceded by escalation of care with admission to ICU.

Although hospice utilization among patients with dementia has been extensively reported $[1,3]$, factors affecting transfer to hospice care have not been examined, to our knowledge, among these patients, once hospitalized. In addition, studies of predictors of hospice care in general did not examine the roles of comorbid conditions, intercurrent organ failure or used life support interventions, with sparse reports to date, which did not address specifically patients with dementia [21].

Our finding of increased odds of hospice discharge with older age among patients with dementia contrasts a recent report by Kiely and colleagues, with the latter showing no impact of age among nursing home residents with advanced dementia in the Boston area [22]. The difference may be related in part to the relatively small sample size of the latter study, different care setting, and focus only on advanced dementia. However, because administrative data do not include information on the severity of dementia, our findings may reflect confounding, with older hospitalizations with dementia being more likely to have more severe disease.

The markedly reduced likelihood of discharge to hospice among minority hospitalizations noted in the present study is consistent with prior reports of disparities in use of hospice services among minority patients, across diagnoses and care settings [23], with increased preference for dying in the hospital among black and Hispanic patients [24]. Our findings contrast reports of adjusted higher odds of hospice care among non-white nursing home residents with advanced dementia [22] and, as noted earlier, the difference may be related to difference of sample size, care setting, and possibly geographical region. The reasons for the noted racial disparities in hospice care remain incompletely understood [23]. The complexities involved in interpreting varying use of hospice care across ra- cial groups are underscored by a recent study by Elliott and colleagues showing that although hospital-based physicians use similar verbal communication when discussing EOL care with black and white patients, they exhibit significantly fewer rapport-building nonverbal cues with the former [25].

The role of health insurance in hospice utilization among hospitalized older adults with dementia has not been previously examined. Lower use of hospice services among patients with traditional Medicare insurance, as compared with those with commercial insurance was previously described in the general population $[26,27]$, as was noted consistently in the present cohort, though the underlying explanations remain elusive [26]. Although charity care is offered by many hospice providers [2], this is not the case for about one in five nonprofit or for-profit facilities [28], which may have contributed to the lower odds of hospice use in the present cohort among those lacking health insurance. However, lack of health insurance was no longer significant predictor of hospice use by the end of the last decade, possibly reflecting improved access for these patients. We found, unexpectedly, that Medicaid insurance was associated with the lowest odds of hospice use, even as compared to those lacking health insurance. Our results contrast a recent report by duPreez and colleagues showing no independent impact of Medicaid insurance on use of hospice among elderly decedents following acute stroke [21]. Although patients with Medicaid insurance are eligible for hospice benefits in Texas, those are restricted to patients with full Medicaid insurance [29]. It is unclear how or whether varying insurance coverage has affected clinicians' behavior related to hospice referral and to what extent our findings reflect statespecific barriers to hospice use. However, our findings represent a target for further study to identify means to improve patients' access.

While presence of non-dementia comorbidities generally increased the odds of hospice discharge in our cohort especially, as expected, among those with concurrent malignancy, comorbid conditions generally did not predict hospice use among decedents following acute stroke [21]. The difference between studies may be related to unmeasured factors between hospitalized patients with dementia, at times with other interim complications vs. those specifically following stroke, as well as different study designs.

The association of organ failure with subsequent hospice care among hospitalized patients has not been previously examined. Our findings suggest that development of even a single acute organ failure, regardless of the affected system, was likely among the key factors in considering transition to hospice care during discussions between clinicians and HCP, with the magnitude of association generally higher than that of comorbid conditions. Although development of respiratory failure doubled the odds of patients' referral to hospice care, those decisions often occurred following institution of invasive mechanical ventilation. Thus, finding that institution of mechanical ventilation was associated independently with increased odds of hospice care highlights gaps in timely effective communication about patients' goals of care, once care was escalated to hospital setting.

Our study quantifies for the first time the increasingly prevalent use of ICU among hospitalized older adults with de- 
mentia who are subsequently discharged to hospice. Although hospice use rose markedly among hospitalized patients, transition to hospice care was increasingly preceded by further, potentially avoidable, escalation of care to ICU, accounting for nearly one in two hospice discharges by the end of the decade. The latter pattern suggests that increasing proportion of key discussions by clinicians about goals of care and options of palliative care likely took place following admission to ICU and, at times, after institution of invasive life support interventions. Indeed, prior study by Rady and colleagues in the general population showed that communication about alternative care goals was uniformly absent prior to transfer to ICU among hospital decedents in a single-center cohort [13]. Admission to ICU and related life-support interventions may meet short- and long-term goals of some patients with dementia, and lack of patient-centered benefits cannot be assumed arbitrarily. However, the present findings underscore the need to effectively incorporate discussions on ICU admission and related interventions vs. alternative care options early in the hospital course.

The study has several strengths, including use of a highquality data set in a state with a diverse population, a robust sample size, and a population-based approach, with the latter allowing transcending local variability in care patterns.

Nevertheless, the interpretation of the present findings should be considered in the context of several limitations. We used a retrospective design and administrative data with their attendant limitations. Although we used code-based approach similar to that of other investigators $[16,17]$, use of ICD-9 codes to identify hospitalizations with dementia may have led to misclassification in some cases. A previous report documented sensitivity and specificity of $85 \%$ and $89 \%$, respectively, for diagnosis of dementia using similar ICD-9 codes [30]. In addition, the severity of dementia could not be determined and it has been suggested that administrative data may underestimate milder stages of dementia [31], related in part to prevalent underestimation of pre-existing cognitive dysfunction in the hospital setting $[32,33]$.

In addition, use of administrative data precluded examination of pre-existing limitations of care among hospitalized patients and their interaction with the identified predictors of hospice discharge. Similarly, the specialties, level of training, and experience of the involved clinicians, though bound to affect the timing and content of communication with HCP and the decision-making by the latter, could not be examined.

Finally, although the present study examined data from a large state with a diverse population, the use of hospice services varies substantially across states [34], as can population characteristics, thus affecting the generalizability of our findings.

In conclusion, decisions about transition to hospice care among hospitalized older adults with dementia appeared generally sensitive to the presence of non-dementia comorbidities and especially to measures of increasing severity of illness. However, even following adjustment for other confounders, disparities in use of hospice services persisted among nonwhite minorities and hospitalizations with non-commercial primary health insurance. In addition, the marked rise in hospice utilization during EOL hospitalizations was tempered by concomitant substantial increase in discharges to hospice only following admission to ICU, suggesting common delays in effective communication with HCP.

The findings of the factors associated with increased hospice use and potentially modifiable barriers can inform policy-, healthcare system-, and clinician-level studies and interventions geared to better align in advance patients' goals of care with subsequent interventions.

\section{Acknowledgments}

I would like to thank Phillip Watkins, MS, for his help with parts of data acquisition and management.

\section{Conflicts of Interest}

The author declares that there are no conflicts of interest regarding the publication of this paper.

\section{Grant Support}

None.

\section{References}

1. Teno JM, Gozalo PL, Bynum JP, Leland NE, Miller SC, Morden NE, Scupp T, et al. Change in end-of-life care for Medicare beneficiaries: site of death, place of care, and health care transitions in 2000, 2005, and 2009. JAMA. 2013;309(5):470-477.

2. NHPCO's Facts and Figures: Hospice Care in America, 2015 Edition. National Hospice and Palliative Care Organization. Available at: http://www.nhpco.org/sites/ default/files/public/Statistics_Research/2015_Facts_Figures.pdf. Accessed February 15, 2016.

3. Mitchell SL, Teno JM, Kiely DK, Shaffer ML, Jones RN, Prigerson HG, Volicer L, et al. The clinical course of advanced dementia. N Engl J Med. 2009;361(16):15291538.

4. Givens JL, Selby K, Goldfeld KS, Mitchell SL. Hospital transfers of nursing home residents with advanced dementia. J Am Geriatr Soc. 2012;60(5):905-909.

5. Mitchell SL, Teno JM, Intrator O, Feng Z, Mor V. Decisions to forgo hospitalization in advanced dementia: a nationwide study. J Am Geriatr Soc. 2007;55(3):432-438.

6. Lamberg JL, Person CJ, Kiely DK, Mitchell SL. Decisions to hospitalize nursing home residents dying with advanced dementia. J Am Geriatr Soc. 2005;53(8):13961401.

7. Coleman EA. Falling through the cracks: challenges and opportunities for improving transitional care for persons with continuous complex care needs. J Am Geriatr Soc. 2003;51(4):549-555.

8. Zilberberg MD, Tjia J. Growth in dementia-associated 
hospitalizations among the oldest old in the United States: implications for ethical health services planning. Arch Intern Med. 2011;171(20):1850-1851.

9. Oud L. Evolving Demand for Critical Care Services for Elderly Adults with Dementia in Texas: A PopulationBased Study. J Am Geriatr Soc. 2016;64(2):432-434.

10. 2012 Alzheimer's disease facts and figures. Alzheimer's Association. Alzheimers Dement. 2012;8(2):87-170.

11. Callahan CM, Tu W, Unroe KT, LaMantia MA, Stump TE, Clark DO. Transitions in Care in a Nationally Representative Sample of Older Americans with Dementia. J Am Geriatr Soc. 2015;63(8):1495-1502.

12. Iwashyna TJ, Ely EW, Smith DM, Langa KM. Long-term cognitive impairment and functional disability among survivors of severe sepsis. JAMA. 2010;304(16):17871794.

13. Rady MY, Johnson DJ. Admission to intensive care unit at the end-of-life: is it an informed decision? Palliat Med. 2004;18(8):705-711.

14. Texas inpatient public use data file. Texas Department of State Health Services, Center for Health Statistics, Austin, Texas (online). Available at: http://www.dshs.state. tx.us/thcic/hospitals/Inpatientpudf.shtm. Accessed November 12, 2015.

15. Oud L, Watkins P. Contemporary trends of the epidemiology, clinical characteristics, and resource utilization of necrotizing fasciitis in Texas: a population-based cohort study. Crit Care Res Pract. 2015;2015:618067.

16. Lagu T, Zilberberg MD, Tjia J, Pekow PS, Lindenauer PK. Use of mechanical ventilation by patients with and without dementia, 2001 through 2011. JAMA Intern Med. 2014;174(6):999-1001.

17. Guerra C, Linde-Zwirble WT, Wunsch H. Risk factors for dementia after critical illness in elderly Medicare beneficiaries. Crit Care. 2012;16(6):R233.

18. Deyo RA, Cherkin DC, Ciol MA. Adapting a clinical comorbidity index for use with ICD-9-CM administrative databases. J Clin Epidemiol. 1992;45(6):613-619.

19. Kumar G, Kumar N, Taneja A, Kaleekal T, Tarima S, McGinley E, Jimenez E, et al. Nationwide trends of severe sepsis in the 21 st century (2000-2007). Chest. 2011;140(5):1223-1231.

20. Bingold TM, Lefering R, Zacharowski K, Meybohm P, Waydhas C, Rosenberger P, Scheller B. Individual Organ Failure and Concomitant Risk of Mortality Differs According to the Type of Admission to ICU - A Retrospective Study of SOFA Score of 23,795 Patients. PLoS One. 2015;10(8):e0134329.

21. duPreez AE, Smith MA, Liou JI, Frytak JR, Finch MD, Cleary JF, Kind AJ. Predictors of hospice utilization among acute stroke patients who died within thirty days.
J Palliat Med. 2008;11(9):1249-1257.

22. Kiely DK, Givens JL, Shaffer ML, Teno JM, Mitchell SL. Hospice use and outcomes in nursing home residents with advanced dementia. J Am Geriatr Soc. 2010;58(12):22842291.

23. Johnson KS. Racial and ethnic disparities in palliative care. J Palliat Med. 2013;16(11):1329-1334.

24. Barnato AE, Anthony DL, Skinner J, Gallagher PM, Fisher ES. Racial and ethnic differences in preferences for end-of-life treatment. J Gen Intern Med. 2009;24(6):695701.

25. Elliott AM, Alexander SC, Mescher CA, Mohan D, Barnato AE. Differences in Physicians' Verbal and Nonverbal Communication With Black and White Patients at the End of Life. J Pain Symptom Manage. 2016;51(1):1-8.

26. Virnig BA, Fisher ES, McBean AM, Kind S. Hospice use in Medicare managed care and fee-for-service systems. Am J Manag Care. 2001;7(8):777-786.

27. Emanuel EJ, Ash A, Yu W, Gazelle G, Levinsky NG, Saynina $\mathrm{O}$, McClellan $\mathrm{M}$, et al. Managed care, hospice use, site of death, and medical expenditures in the last year of life. Arch Intern Med. 2002;162(15):1722-1728.

28. Aldridge MD, Schlesinger M, Barry CL, Morrison RS, McCorkle R, Hurzeler R, Bradley EH. National hospice survey results: for-profit status, community engagement, and service. JAMA Intern Med. 2014;174(4):500-506.

29. Medicaid for the Elderly and People with Disabilities Handbook. Revision: 13-4. December 1, 2013. Texas Health and Human Services Commission. Available at: https://www.dads.state.tx.us/handbooks/mepd/ A/A-5000.htm. Accessed March 7, 2016.

30. Taylor DH, Jr., Ostbye T, Langa KM, Weir D, Plassman BL. The accuracy of Medicare claims as an epidemiological tool: the case of dementia revisited. J Alzheimers Dis. 2009;17(4):807-815.

31. Bynum JP, Rabins PV, Weller W, Niefeld M, Anderson $\mathrm{GF}, \mathrm{Wu} \mathrm{AW}$. The relationship between a dementia diagnosis, chronic illness, medicare expenditures, and hospital use. J Am Geriatr Soc. 2004;52(2):187-194.

32. Pisani MA, Redlich CA, McNicoll L, Ely EW, Friedkin RJ, Inouye SK. Short-term outcomes in older intensive care unit patients with dementia. Crit Care Med. 2005;33(6):1371-1376.

33. Pisani MA, Redlich C, McNicoll L, Ely EW, Inouye SK. Underrecognition of preexisting cognitive impairment by physicians in older ICU patients. Chest. 2003;124(6):2267-2274.

34. Wang SY, Aldridge MD, Gross CP, Canavan M, Cherlin E, Johnson-Hurzeler R, Bradley E. Geographic Variation of Hospice Use Patterns at the End of Life. J Palliat Med. 2015;18(9):771-780. 